\title{
NEWLY DEVELOPED INJECTION MODE OF PLS
}

\author{
M. G. Kim, J. Choi, J. Y. Huang and E. S. Park \\ Pohang Accelerator Laboratory, Pohang, Gyungbuk, 790-784 Korea
}

\begin{abstract}
At the Pohang Light Source, $2 \mathrm{GeV}$ electron beam had been injected to the storage ring and increased to $2.5 \mathrm{GeV}$ using an energy ramping system previously. This ramping process was regarded as a source of closed orbit distortions. The successful upgrade of injection power of the PLS linear accelerator has enabled to develop the full energy injection mode, where $2.5 \mathrm{GeV}$ electron beam is injected and synchrotron radiation is provided to the photon beamlines without the ramping process. With the constraints of preserving the so called "golden orbit" and parameters something like betatron tunes, new optimized sets of magnetic fields are searched for, taking into account of possible septum magnetic field leakage. The observed results and improvements after the newly employed injection mode are reported.
\end{abstract}

\section{INTRODUCTION}

The Pohang Light Source (PLS) consists of a linear accelerator and a storage ring. The linear accelerator used to be a $2.0 \mathrm{GeV}$ machine. But since the storage ring is capable of storing $2.5 \mathrm{GeV}$ beam, energy ramping in the storage ring was used.

Obviously one of the primary requirements from users of third generation synchrotron light sources is the stringent transverse stability of the X-ray beam emitted from the bending magnets and insertion devices. However, a long-term drift of the closed orbit is routinely observed in PLS. Between each beam injection occurring in every 12 hours, the closed orbit is drifted up to $20-160 \mu \mathrm{m}$. Since the horizontal beam size is about $200 \mu \mathrm{m}$, this drift corresponds to $20-80 \%$ of the beam size, which needs to be corrected to less than $10 \%$. Unfortunately, a part of this drift was found to come from the energy ramping procedure.

The storage ring has been operated at $2.5 \mathrm{GeV}$ since 1999 by acceleration (energy ramping) procedure of the stored beam. We found that the acceleration procedure was one of the sources of long-term orbit drift. So we tried to inject $2.5 \mathrm{GeV}$ full energy beam without using any acceleration procedure in the storage ring. This paper will describe problems caused by the acceleration process in the storage ring, and their cure by full energy injection.

\section{ACCELERATION PROCESSES}

Basically the ramping procedure simply consists of increasing the currents of the magnet power supplies (MPS) step by step. But the real procedure is not easy in order to keep the storage ring optics unchanged. The relation of the MPS current to the corresponding magnet current is not linear. In the normal operating region near $2.0 \mathrm{GeV}$, it is almost linear, but outside that region the discrepancy grows. Since the discrepancy is different for different magnets, even if all the MPS currents are increased by the same percentage, the real magnetic fields do not change correspondingly and thus the linear optics such as betatron tune is distorted. Even though the amount of distortion in each step is very small, if the ramping procedure continues this way, the betatron tune shifts keep growing and finally the beam blows up. Therefore we tried to keep the betatron tunes same in each step. For this purpose, all MPS should not change by the exactly same percentage, but each MPS current should be fine tuned with respect to the basic percentage was chosen to be $0.3 \%$. In a number of machine studies, we obtained data of appropriate MPS current setting values to keep the betatron tunes same in each step. In order to be sure of safe and stable ramping, we chose the conservative value $0.3 \%$.

\section{Ramping/Degaussing}

We accelerated the stored beam to $2.5 \mathrm{GeV}$ with ramping/degaussing process from 1999 to 2001 . There are several control steps in the process:

- $2.5 \mathrm{GeV}$ stored beam dump down.

- Degaussing all magnets for $2.0 \mathrm{GeV}$ beam injection.

- $2.0 \mathrm{GeV}$ beam injection.

- Acceleration to $2.5 \mathrm{GeV}$.

- Set golden orbit for user service

After the injection, we found routinely long-term orbit drift above160 $\mu \mathrm{m}$ during user service. (See Fig. 1).

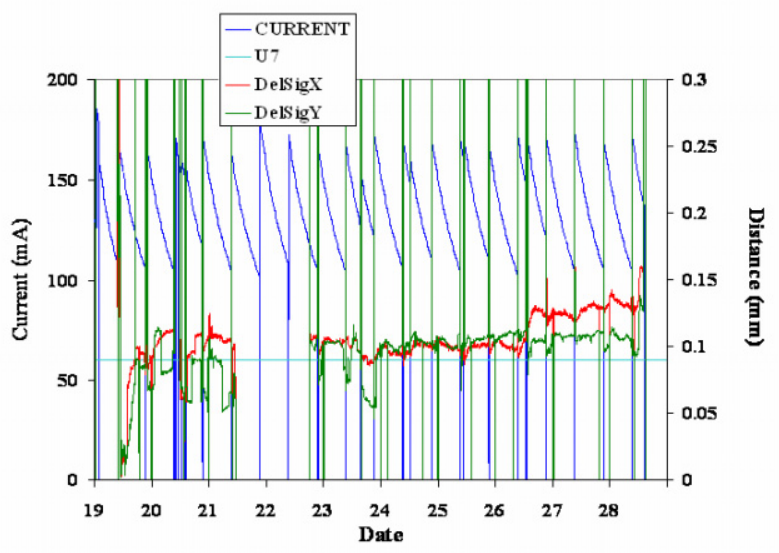

Fig. 1 RMS change during user service run with ramping/ degaussing process for 10 days.

In PLS, one user service run is normally 10 days. Therefore it is the primary job to keep the orbit stable for 10 days. But the ramping/degaussing procedure gave a perturbation to the orbit stability. Because of the degaussing, the magnet cooling system had sudden 
chaotic effect of temperature fluctuation, which caused small perturbation to the beam orbit right after the injection/ramping procedure. Obviously, this perturbation vanishes after a long enough time, but it is preferable to eliminate the perturbation. And the ramping process had another problem; it gave a big load to control system and thus there were some control faults in each user service run.

To solve these problems, we separated the ramping control system from the main control system and used ramping/de-ramping process beginning from May 2001.

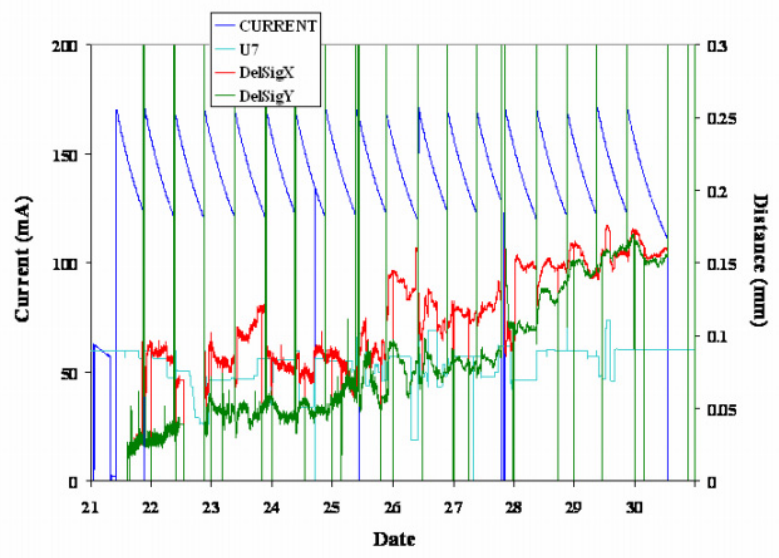

Fig. 2 RMS change during user service run with ramping/ de-ramping process

\section{Ramping/De-ramping}

The new energy ramping system from $2.0 \mathrm{GeV}$ to 2.5 $\mathrm{GeV}$ was installed and used for regular beam injections from May 2001 to Oct. 2002. De-ramping capability from $2.5 \mathrm{GeV}$ to $2.0 \mathrm{GeV}$ was added in the new system to reduce the thermal variation in the magnet cooling system. So we did not dump down the $2.5 \mathrm{GeV}$ stored beam before the injection of $2.0 \mathrm{GeV}$ beam. There are several control steps in the process:

- Deceleration of the stored beam to $2.0 \mathrm{GeV}$.

- Set injection orbit for $2.0 \mathrm{GeV}$ beam injection.

- $\quad 2.0 \mathrm{GeV}$ beam injection.

- Set intermediate orbit for acceleration.

- Acceleration to $2.5 \mathrm{GeV}$.

- $\quad$ Set golden orbit for user service.

Table 1: Control faults and injection time

\begin{tabular}{|c|c|c|}
\hline Injection Mode & $\begin{array}{c}\text { Fault number } \\
\text { of control/run }\end{array}$ & $\begin{array}{c}\text { Injection time } \\
\text { (minute) }\end{array}$ \\
\hline $\begin{array}{c}\text { Ramping/old } \\
\text { control system }\end{array}$ & 4.0 & $20 \sim 15$ \\
\hline $\begin{array}{c}\text { Ramping/separated } \\
\text { control system }\end{array}$ & 1.2 & $15 \sim 10$ \\
\hline $\begin{array}{c}\text { Full energy } \\
\text { injection }\end{array}$ & 0.3 & $8 \sim 5$ \\
\hline
\end{tabular}

With the separated ramping control system, the number of control faults was reduced (See table 1).

But the orbit drift still existed (See Fig. 2). This is because the cooling temperature fluctuation still existed in ramping/de-ramping process. Furthermore the hysteresis of the magnets could not be eliminated in the process. So the orbit drift caused by cooling temperature fluctuation still remained as a problem to be solved.

\section{FULL ENERGY INJECTION}

We concluded that the injection process should be simplified and thus we tried the full energy injection of $2.5 \mathrm{GeV}$ beam.

\section{Linac Upgrade}

The linear accelerator of PLS had been operated continuously by 11 modules, klystron-modulator system (MK) as an injector since December 1994. For $2.0 \mathrm{GeV}$ operation, the required microwave output power from each MK system is approximately $45 \mathrm{MW}$ average at a 10 $\mathrm{Hz}$ repetition rate. For the more stable operation, the 12th MK module was installed and has been operated since 1999. This has contributed to an improvement of the linac energy margin.

The MK12 module consists of two accelerating columns supplied with RF power by one klystron of 80 MW peak, an in-house made modulator of 200 MW peak, and one pulse compressor. The auxiliary systems such as vacuum and cooling water were established by extending the existing system. At commissioning, we obtained the maximum beam energy of about $147 \mathrm{MeV}$. One of the modulators has been running at $17 \mathrm{kV}$ without RF power as it had to keep a spare modulator. This K\&M system is kept at normal operational status throughout the whole year, except for the regularly scheduled maintenance periods.

Full energy of the linac has been raised continuously by various fine tunings like upgrade of IPA controller and cooling system. In November 2002, we achieved stable $2.5 \mathrm{GeV}$ electron beam at the end of the linac for full energy injection.
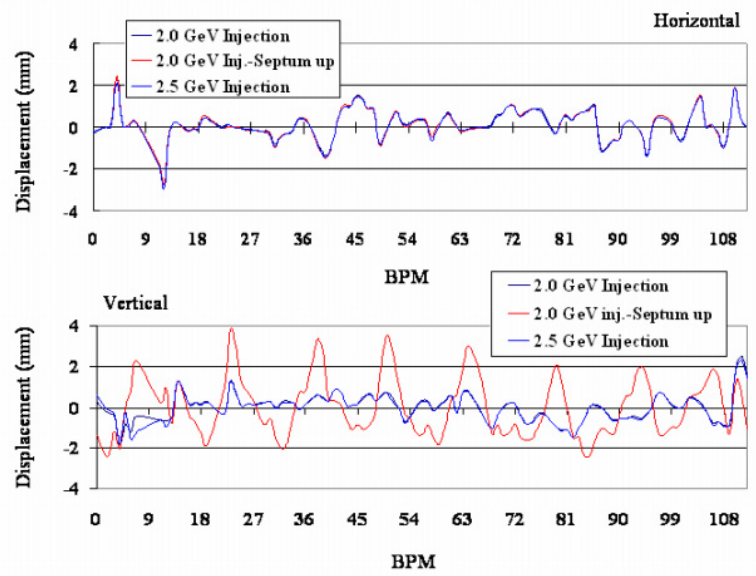

Fig. 3 Orbit correction due to the leakage filed of septum magnet. 


\section{Effect of the Leakage Field of Septum Magnet}

In PLS, a Lambertson type septum magnet is used for injection from the linear accelerator to the storage ring. It bends the injected beam from the beam transfer line vertically and injects it horizontally to the storage ring. In the new $2.5 \mathrm{GeV}$ injection circumstance, the septum magnet showed some leakage field, which can cause additional vertical orbit distortion (see Fig. 3). But it was found that the leakage filed is not strong enough and thus the orbit correction utility of PLS can correct the orbit without any problem. Hence the septum magnet leakage field is not any problem for keeping golden orbit for users.

\section{Full Energy Injection}

Since November 2002, full energy injection has been operated successfully. The most important outcome of this full energy injection mode is the improvement of the orbit stability. Fig. 4 shows the reduction of the orbit drift, especially in the horizontal plane (from $160 \mu \mathrm{m}$ to $20 \mu \mathrm{m}$ )

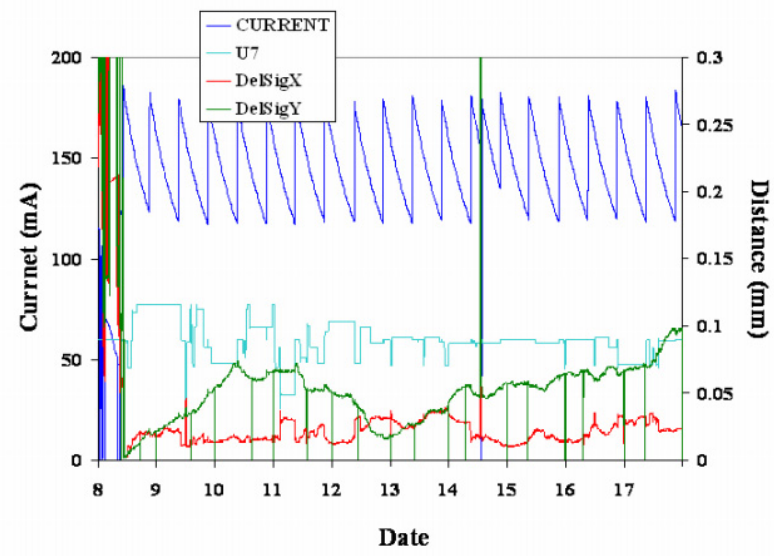

Fig. 4 RMS change with full injection mode during 10 days. Blue line shows stored beam current, sky blue line shows gap of insertion device, red line is horizontal RMS, green line is vertical RMS.

By reducing the orbit drift caused by energy ramping, we can focus more on other sources of orbit distortion such as gap variations of insertion device (see also Fig. 4) or effect of stored beam current (see Fig. 5). Fig. 5 shows the orbit distortion at each injection. The variances of the orbit deviations from the orbit right after every injection suggest a strong dependency of the closed orbit distortions on the stored beam current as shown by the red and blue peaked lines, although it is not easy to thoroughly sort out all the errors involved in measuring the orbit distortions. In an operational point of view the figure shows that the $10 \%$ beam size stability requirement was approximately achieved by the newly developed fullenergy injection mode.

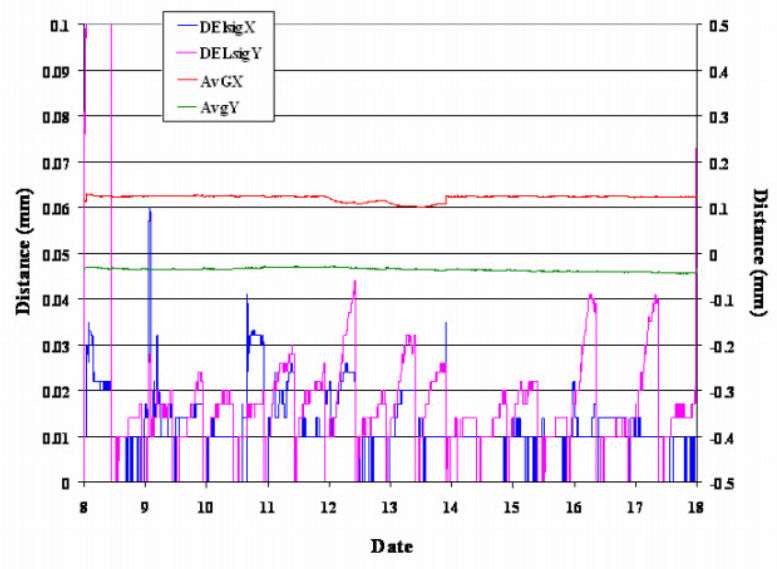

Fig. 5 RMS change in every injection about 12 hours

\section{SUMMARY}

By maximizing the capability of the PLS linear accelerator, it was possible to inject $2.5 \mathrm{GeV}$ beam to the storage ring. This full energy injection improved the orbit stability. At the moment, PLS is using the full energy injection successfully.

\section{REFERENCES}

[1] K. Kim, K. Shim, J. Choi, M. Cho, W. Namkung and I. Ko, Jpn. J. Appl. Phys., Vol. 39, Oct. 2000, p.6094.

[2] 1998, 2000 and 2001 Pohang Accelerator Laboratory Annual Report.

[3] J. Huang, S. Park, W. Hwang, D. Kim and S. Nam, "Improved Closed Orbit Measurement System for PLS,” PAC2001, Chicago, May 2001, p. 1384. 